Original Research Paper

\title{
The Criterion for Determining the Ultimate Pullout Capacity of Plate Anchors in Clay by Numerical Analysis
}

\author{
Haixiao Liu, Fangmei Su and Zhou Li \\ School of Civil Engineering, Tianjin University, Tianjin, China
}

Article history

Received: 11-12-2014

Revised: 23-12-2014

Accepted: 27-12-2014

Corresponding Author:

Haixiao Liu,

School of Civil Engineering,

Tianjin University, Tianjin,

China

Email: liuhx@tju.edu.cn

\begin{abstract}
The Ultimate Pullout Capacity (UPC) is an important index in designing and analyzing plate anchors. However, there are still uncertainties in determining the UPC through the load-displacement curves calculated from numerical analysis. The review on the existing criteria for determining the UPC also demonstrates that there has not been a generally accepted criterion and most existing criteria are not only uncertain but also irrational. Considering that there are still divergences and confusions in determining the UPC and specific studies are rare, further investigations are needed to clarify this basic but important topic before analyzing the UPC and the performance of plate anchors. The present work just emphasizes on the criterion for determining the UPC of plate anchors in clay. After a review of current studies on this topic, the maximum resistance criterion based on large deformation finite element analysis is recommended to determine the UPC of plate anchors, which accords with two rules. The first, a deterministic value of UPC can be obtained by using the criterion. The second, the criterion must be rational which means that it implies clear and right physical meaning and can be generally applied. This criterion is validated firstly by three model tests and then applied to rectangular and circular plate anchors at different embedment depths in both uniform and linear clays to calculate the bearing capacity factors.
\end{abstract}

Keywords: Plate Anchor, Ultimate Pullout Capacity, UPC, Numerical Analysis, Large Deformation, Clay

\section{Introduction}

Plate anchors are widely used in deepwater mooring systems to efficiently provide the pullout capacity by deeply embedding in the seabed. Due to different installation techniques, this type of anchor can be more often distinguished as the drag embedment plate anchor and the suction embedded plate anchor. The plate anchor presents macroscopically tabulate with very large ratio of longitudinal and latitudinal sizes to thickness and with different shapes such as rectangular and circular ones. Attributed to the large plate area normal to the loading, the plate anchor can provide huge capacity even to 100 times of its weight.

Lots of numerical studies have been conducted to explore the installation techniques and to examine the working performance of plate anchors, especially the Ultimate Pullout Capacity (UPC) of the plate anchor with various shapes in different soils. The UPC is an important index in designing and analyzing plate anchors. To obtain a safe as well as economical anchor, it is vital to have an accurate estimation of the UPC by numerical modeling. However, the authors' practice has recognized that there are uncertainties in determining the UPC by numerical analysis. Figure 1 illustrates two typical load-displacement curves in numerical modeling, in which the left exhibits more ideal in determining the UPC because a clear plateau that corresponds to the UPC arises, while the right seems indistinct for determining the UPC because the load always increases with displacement and a plateau can never be found. In reality, curves like the right are more common among load-displacement curves through numerical modeling, which consist of generally three phases including the elastic, yield and plastic flow phases divided by the straight, tortuous and straight segments correspondingly and in many cases the load-displacement curve shows a tortuous shape in the whole process. This means that a 
definite criterion for determining the UPC through the load-displacement curve must be established first before analyzing the UPC and the performance of the anchor.

Although the literature in numerically analyzing the performance of anchors is rich, only a few of studies that clearly pointed out the criterion for determining the UPC, which will be discussed in detail in the following section. The review on the existing criteria for determining the UPC demonstrates that, there has not been a generally accepted criterion and most existing criteria are not only uncertain but also irrational. Therefore, a real criterion that can be adopted to completely exclude uncertainties meanwhile with clear physical meaning is important to be developed, since that the criterion is the basis for analyzing the UPC and the performance of plate anchors.

Two types of complete load-displacement curves of the anchor in experiments are shown in Fig. 2, in which each curve can be clearly divided into two regions by a peak. At the first region, the load increases with displacement, while the load decreases with displacement at the second region. Depending on different structures or soils, the increase or decrease rates of the load may be

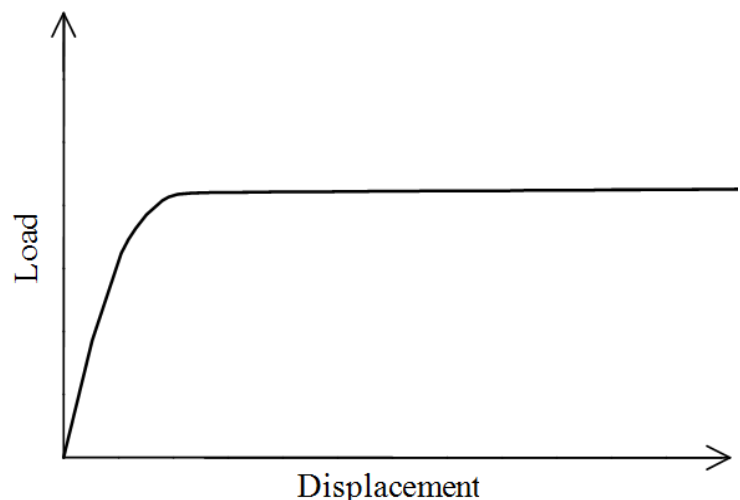

(a) different. Anyhow, a peak is always existent. Obviously, the peak value denotes the maximum bearing capacity of the anchor under monotonic loading and in physical meaning it should be identical to the UPC. On the other hand, if the peak value of the load-displacement curve is chosen as the UPC, then the UPC can be determined easily and uniquely.

Note that most criteria for determining the UPC are based on small deformation numerical analysis, from which a complete load-displacement response cannot be obtained to find the peak value. In a small deformation analysis, if the anchor displacement is relatively large, a non-convergent solution may be induced and the calculation would be interrupted due to severe mesh distortions. During the whole process of computation, a declining tendency of pullout force as that in model tests can never appear. Therefore, small deformation numerical methods are not appropriate to capture the complete load-displacement response of plate anchors. Recently, researchers have realized the drawback of small deformation analysis and turned to large deformation techniques to analyze large deformation problems, such as the anchor related ones.

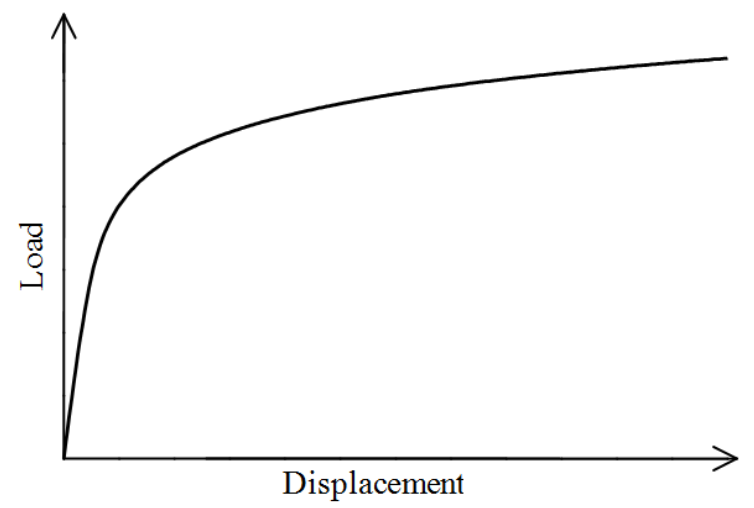

(b)

Fig. 1. Two typical load-displacement curves in numerical modeling

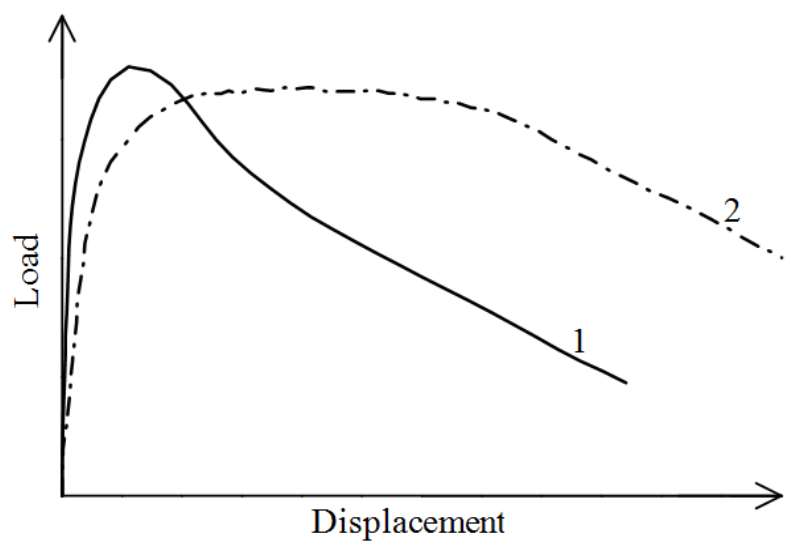

Fig. 2. Complete load-displacement curves of the anchor in experiments 
Considering that there are still divergences in determining the UPC and specific studies are rare, further investigations are needed to clarify this basic but important topic. The present work just emphasizes on the criterion for determining the UPC of plate anchors in clay. After a review of current studies on this topic, the criterion for determining the UPC based on large deformation finite element analysis is recommended, which is deterministic and conceptually rational. This criterion is then applied to rectangular and circular plate anchors at different embedment ratios in both uniform and linear clays to calculate the bearing capacity factors.

\section{Current Criteria Used in Determining the UPC}

The existing methods for determining the UPC of anchors in numerical study, which can be found from publications, are classified as the plateau criterion, the linearly increasing criterion, the double tangent intersection criterion, the function fitting criterion, the specified displacement criterion, the K4 criterion and the maximum resistance criterion.

\section{The Plateau Criterion}

This method postulates a final plateau in the loaddisplacement curve, at which the load sustains or slightly increases while the movement grows rapidly. A horizontal straight line is used to fit the plateau, at which the value of load is the UPC (Fig. 3a). This criterion was ever adopted by O'Neill et al. (2003; Yang et al., 2010; Sahoo and Kumar, 2013) in numerical studies. Note that although Chen et al. (2013) employed large deformation finite element analysis to study the UPC of square anchors embedded in uniform clay, this criterion was also adopted by them. However, not all loaddisplacement curves have a plateau. For those curves without a distinct plateau, this method is not applicable. For those with a mild slope, the plateau value is somehow judgmental instead of rigorous.

\section{The Linearly Increasing Criterion}

The linearly increasing criterion considers the UPC as the resisting force at which the load begins to linearly increase with the displacement. As illustrated in Fig. 3b, at the final portion of the curve, when the displacement increases, the resisting force also increases remarkably and a plateau could not be found. To apply this method, a straight line is used to fit the final segment of the curve and the load at the intersection is regarded as the UPC. Neeley et al. (1973; Ghaly, 1997) adopted this approach to determine the UPC of anchors. However, the final segment of the curve is not strictly linear and the result would depend on the relevant factor of the fitting curve.

\section{The Double Tangent Intersection Criterion}

This approach defines the UPC as the resisting force at the intersection of two tangent lines, approximating the initial elastic portion and the final plastic portion of the load-displacement curve (Fig. 3c). Boushehrian et al. (2009) used this criterion to analysis the UPC of shallow foundations. Actually, this method was also adopted by many researchers for simply acquiring the UPC of anchors. This criterion greatly depends on judgment and the scale of the curve owing to the curve fitting approach adopted.

\section{The Function Fitting Criterion}

This criterion fits the load-displacement curve with certain function and the UPC is derived through mathematical modification. Hanna et al. (2007) investigated the load-displacement curve derived by Ghaly et al. (1991) in model tests. A hyperbolic curve was adopted to fit the measured result, which is Equation 1:

$$
Q=1 /(a+b / S)
$$

where, $Q$ is the resisting force of anchor; $S$ is the displacement; $a$ and $b$ are the fitting coefficients. The UPC of anchor could be calculated as $Q_{u l t}=1 / a$, which is the limit of the function $Q$.

Obviously, this criterion depends on the function fitted and the result is not unique with different fitting coefficients.

\section{The Specified Displacement Criterion}

This method considers the UPC as the load when the movement of anchor exceeds a certain value. This is because large movement is usually not tolerable for structures supported by anchors.

Vesic (1973) defined the ultimate load at the point of break of the load-displacement curve in a $\log / \log$ plot, which requires displacement as much as $50 \%$ of the foundation size. From the practical point of view, unless a clearly defined unlimited load (i.e., general shear failure) could be observed earlier, the UPC would be regarded as the load when reaching a limit of critical settlement, such as $10 \%$ of the footing depth. In the small deformation analysis by Song et al. (2008), the ultimate capacity was assumed to be at a given displacement value. For circular anchors, the specified displacement is $25 \%$ of the anchor diameter. Mistri and Singh (2011) conducted numerical analysis on plate anchors. The failure load was regarded as the resistance when the displacement of anchor reached $2.5 \%$ of the embedment depth.

As pointed out by Song et al. (2008), with this criterion, the anchor capacity may be lower than the true capacity because of its dependence on the soil stiffness. 
If the soil stiffness is small, the load of anchor would reach a stable value at a large displacement which may exceed the specified displacement. Moreover, the specified displacement is subjective and somehow arbitrary. Different ultimate capacities would be obtained with different specified displacements.

\section{The K4 Criterion}

Rowe and Davis (1982) recommended the K4 criterion to determine the UPC of anchor. The K4 failure load is defined as the resistance when the stiffness of the soil has reduced to one quarter of its initial elastic stiffness. They found out that owing to contained plastic flow, before failure occurred, the deformation could be quite significant. Thus, for practical reasons, the UPC would be considered to have occurred before the true collapse load was reached. Fig. $3 d$ shows the way to obtain the UPC using the K4 criterion in a loaddisplacement curve. This method was also adopted by Fahmy et al. (2013) in numerical study.

The purpose of K4 criterion is identical to that of specified displacement criterion, which is to avoid overwhelming deformation of structure. Comparing with the specified displacement criterion, the $\mathrm{K} 4$ criterion is more flexible since it accounts for the initial elastic deformation of the anchor. This method is more likely an empirical result in terms of the multiple to be used. However, the so-called initial elastic portion of loaddisplacement response is usually not perfectly linear. To apply this criterion, curve fitting is needed. Using different relevant factors would obtain different results. Moreover, there is not a reliable physical meaning with the multiple used in the $\mathrm{K} 4$ criterion.

\section{The Maximum Resistance Criterion}

Wang et al. (2009) investigated the UPC of plate anchors in various shapes based on a large deformation numerical technique known as the Remeshing and Interpolation Technique with Small Strain (RITSS) method. The maximum loading during the pullout process of anchor is regarded as the UPC. As mentioned earlier, the large deformation analysis can well simulate the whole pullout process and obtain a complete loaddisplacement curve of plate anchors. The compensation is that more computing time is needed compared with small deformation methods.

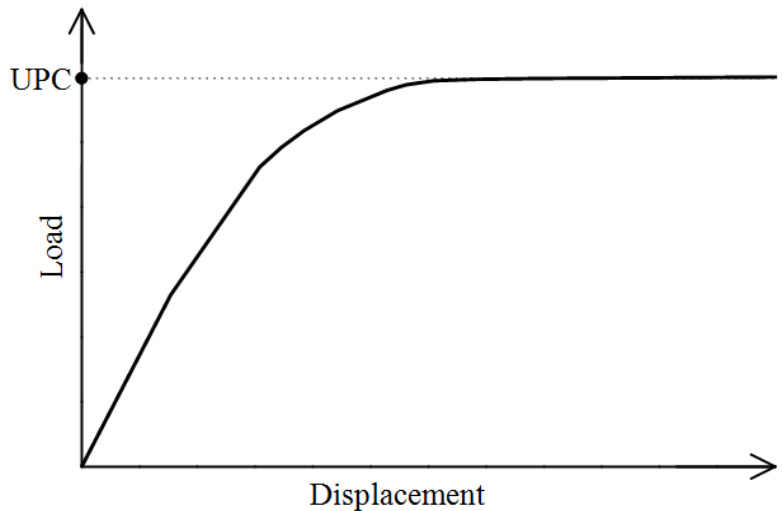

(a)

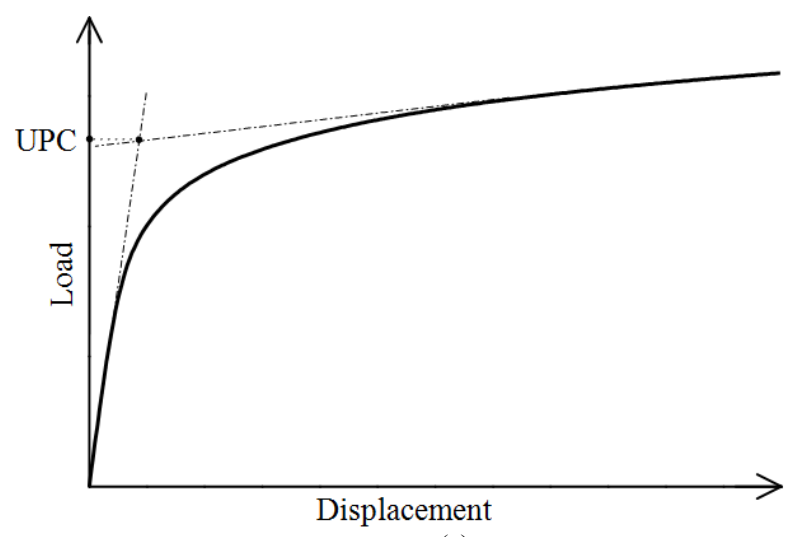

(c)

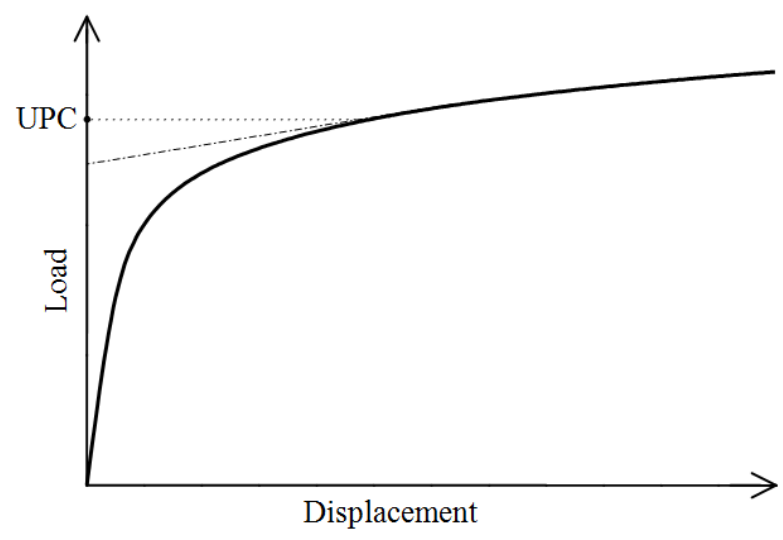

(b)

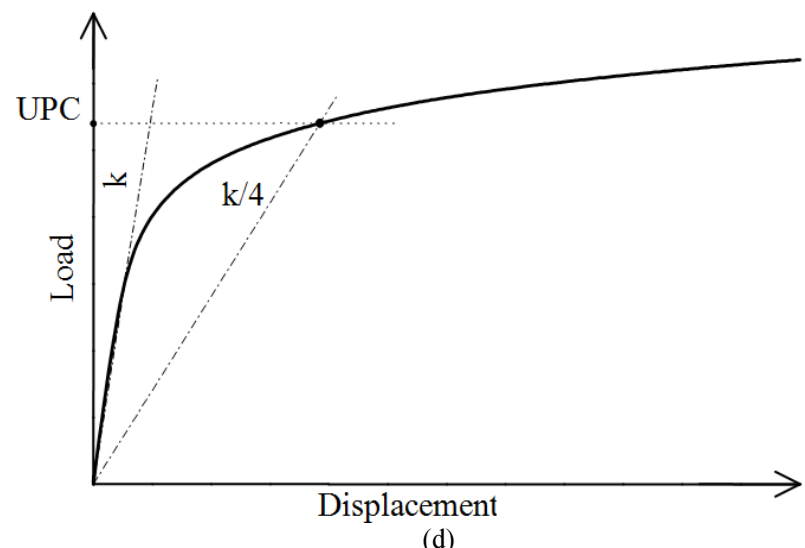

Fig. 3. Different criteria for determining the UPC (a) The plateau criterion (b) The linearly increasing criterion (c) The double tangent intersection criterion (d) The K4 criterion 


\section{The Method for Determining the UPC Using Large Deformation FE Analysis}

\section{Basic Consideration}

In concept, the true UPC is the maximum resisting force during the pullout process of plate anchors. This was also admitted by proposers of the $\mathrm{K} 4$ criterion and the specified displacement criteria. However, for serviceable reasons, the UPC is determined at the point before the peak resistance with different approaches in the two criteria.

Note that except the maximum resistance criterion, all criteria for determining the UPC are based on small deformation analysis. If calculate a load-displacement curve like in Fig. 1a, then the plateau criterion can be adopted to obtain a precise value of UPC. However, Small deformation analysis will be more common to produce the type of load-displacement curves like in Fig. 1b. Small deformation methods cannot correctly reflect the complete load-displacement response when the anchor is greatly mobilized. Therefore, any criteria based on small deformation analysis, which try to determine the UPC according to a small fraction of the load-displacement response, generally cannot produce a rational and deterministic value of UPC, as introduced earlier. It is also noted that only Wang et al. (2009) adopted the maximum resistance criterion to determine the UPC of plate anchors by using the large deformation method (RITSS). Being an interesting comparison, although Chen et al. (2013) also utilized the large deformation method known as coupled Eulerian-Lagrangian technique, the plateau criterion was still adopted to determine the UPC of plate anchors. This seems that the maximum resistance criterion is not definitely adopted even if the large deformation analysis is performed.

Anyway, there are still divergences and confusions in determining the UPC of plate anchors. In the authors' opinion, two rules must be conformed to propose a right criterion. The first, a deterministic value of UPC can be obtained by using the criterion. The second, the criterion must be rational which means that it implies clear and right physical meaning and can be generally applied. Considering the two rules, the maximum resistance criterion based on large deformation analysis is recommended to determine the UPC of plate anchors. In the present work, the Coupled Eulerian-Lagrangian (CEL) technique, which is incorporated in the software ABAQUS, is used in the large deformation analysis.

\section{Validation by Experiments}

To examine the CEL technique as well as the criterion recommended in the present study, three numerical models are built and compared with model tests, including: (1) square anchor in uniform clay; (2) square anchor in linear clay; and (3) circular anchor in linear clay.

\section{(1) Square anchor in uniform clay}

The numerical model is constructed completely simulating the vented square anchors in the model tests conducted by Singh and Ramaswamy (2008). The square plate anchor has the width $(B)$ of $50 \mathrm{~mm}$ and the thickness $(t)$ of $6 \mathrm{~mm}$. The plate is modeled as a discrete rigid solid and meshed with eight-node Lagrangian brick elements. Cases with H/B varying from 1 to 10 are examined, where $\mathrm{H}$ denotes the embedment depth. The soil is modeled as an Eulerian domain and meshed using an eight-node Eulerian brick with reduced integration. The clay has uniform undrained shear strength $\left(s_{u}\right)$ of 2.8 $\mathrm{kPa}$ and a saturated unit weight $(\gamma)$ of $16.61 \mathrm{kN} / \mathrm{m}^{3}$. The clay under undrained conditions is regarded as an elasticperfectly plastic material obeying the Tresca yield criterion. The geostatic stress is generated by taking $K_{0}=$ 1 , where $K_{0}$ denotes the coefficient of earth pressure. The Poisson's ratio is set to 0.495 . The contact between the soil and the plate is assumed to be frictionless. Since the rigidity index $E / s_{u}$, where denotes the Young's modulus of the soil, was not given by the model tests, $E / s_{u}=100$, 300 and 500 are assigned in the numerical modeling. By taking advantage of the symmetry, only a quarter of the square anchor and soil domain is modeled, as illustrated in Fig. 4. The bottom of the model is fixed. Symmetric boundary conditions are imposed on the two planes of symmetry by prescribing zero flow velocity normal to these planes. A convergence study is conducted on mesh size and pullout rate of anchor in order to achieve a balance between the accuracy and computational efficiency. The minimum mesh size of soil around the moving path of anchor is $0.025 \mathrm{~B}$ and the pullout rate of anchor is $0.2 \mathrm{~B} \mathrm{~m} / \mathrm{s}$. The calculated results are processed in the way as adopted by Singh and Ramaswamy (2008). As proposed by Singh and Ramaswamy (2008), the vertical pullout capacity of the plate anchor embedded in saturated clay can be expressed as Equation 2:

$Q_{u}=Q_{0}+Q_{s}+W_{a}$

where, $Q_{0}$ is the net ultimate pullout capacity; $Q_{s}$ is the soil suction effect; $W_{a}$ is the self weight of anchor and the net ultimate pullout capacity can be expressed as Equation 3:

$$
Q_{0}=A\left(s_{u} N_{c}+\gamma H\right)
$$

Since an immediate breakaway condition was set up between anchor and soil in the model tests, the suction effect can be neglected. Then the bearing capacity factor is Equation 4:

$$
N_{c}=\frac{Q_{u}-\gamma H-W_{a}}{A s_{u}}
$$




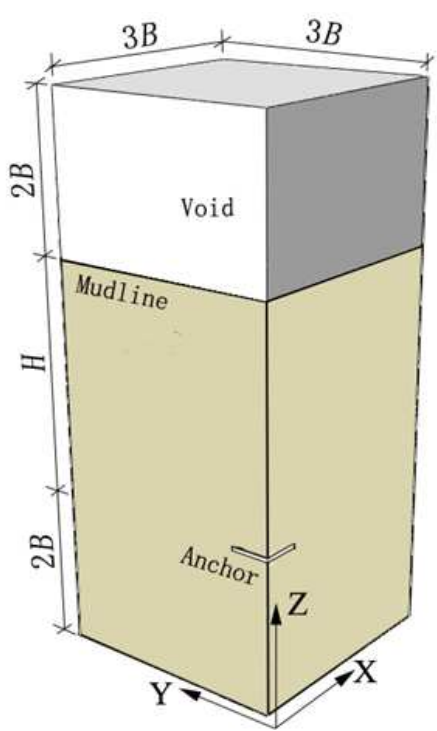

(a)

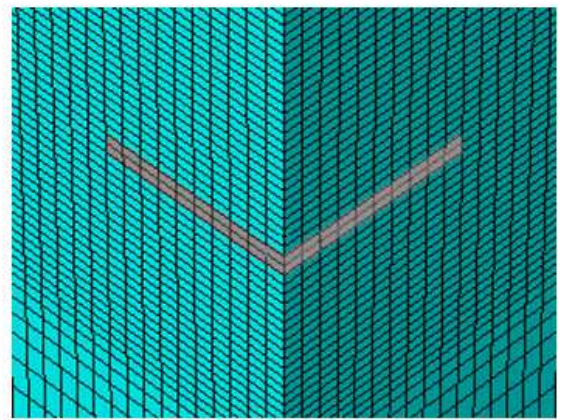

(b)

Fig. 4. Numerical model (a) Global numerical model (b) Meshing details around the anchor

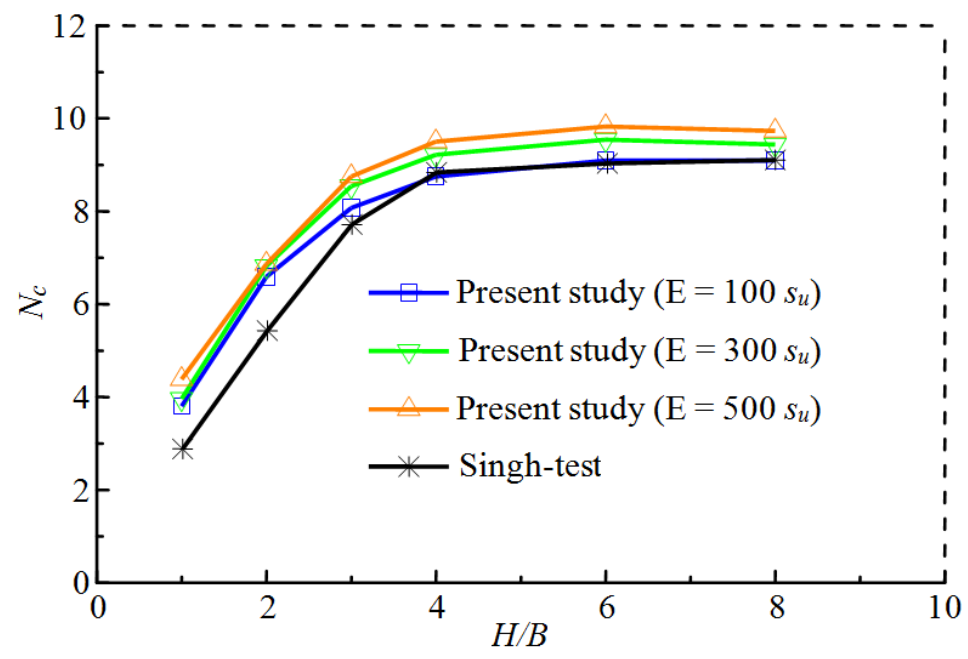

Fig. 5. Comparison of the bearing capacity factor of the square anchor in uniform clay

The comparison of the bearing capacity factor of the square anchor in uniform clay is presented in Fig. 5. It is observed that the trend of the calculated results is generally identical with that of measured data. The value $N_{c}$ of increases rapidly before the embedment depth of 4 $\mathrm{B}$, while becomes nearly stable after $4 \mathrm{~B}$. There is a better agreement between the calculated results with $E / s_{u}$ $=100$ and the measured data especially after the embedment depth of $3 \mathrm{~B}$.

\section{(2) Square anchor in linear clay}

The numerical model is constructed simulating the centrifuge tests by Chen et al. (2014). The centrifuge tests were conducted under an acceleration of $50 \mathrm{~g}$ on anchors with the embedment ratio varying from 0.54 to 2.86 and under an acceleration of $100 \mathrm{~g}$ on anchors only embedded at the depth of $2.86 \mathrm{~B}$. The square plate anchor has the width (B) of $4 \mathrm{~m}$ and the thickness (t) of $0.2 \mathrm{~m}$. The clay has a linear undrained shear strength of $s_{u}=1.34 \mathrm{z}$, where $\mathrm{z}$ denotes the soil depth and the unit of $s_{u}$ is $\mathrm{kPa}$ and a saturated unit weight $(\gamma)$ of $16.07 \mathrm{kN} / \mathrm{m} 3$. Since the rigidity index $E / s_{u}$ was not given by the centrifuge tests, $E / s_{u}=100,300$ and 500 are assigned in the numerical modeling. Cases with $H / B=0.54,1.05$, 2.13 and 3.5 are examined. Considering that different soil and anchor are used here, the parameter study on mesh size and pullout rate as performed earlier is also necessary to balance the accuracy and computational 
efficiency. The minimum mesh size of soil around the moving path of anchor is $0.025 \mathrm{~B}$ and the pullout rate of anchor is $0.025 \mathrm{~B} \mathrm{~m} / \mathrm{s}$.

Other conditions including the yield criterion, values of $K_{0}$ and the Poisson's ratio, the contact between soil and anchor and the boundary condition are all the same with the previous model.

In the CEL technique, nodes are fixed in the space throughout the analysis and do not follow the material movement. A user-defined subroutine was developed to attach the soil strength to material so that the soil strength can be updated with the material flow. Figure 6 shows the contour of linear soil strength $(\mathrm{H} / \mathrm{B}=1)$ during the anchor pullout process, in which Fig. 6a denotes the contour before the pullout process and Fig. $6 \mathrm{~b}$ denotes the contour at the displacement 0.25 B. With this implementation, the distribution of soil strength would be more reasonable and accurate.

The centrifuge test data of Chen et al. (2014) and the present numerical results are plotted together in Fig. 7, in which the normalized pullout capacity of anchor is calculated as Equation 5:

$$
N=\frac{Q_{u}-W_{a}}{A s_{u}}
$$

It is observed that on average, values of the capacity factor at the rigidity index of $100 s_{u}$ agree best with the model tests, where the maximum difference is $8.1 \%$ higher than the model test at the embedment depth of $0.54 \mathrm{~B}$ and the minimum difference is $1.1 \%$ lower than the model test at the embedment depth of $2.86 \mathrm{~B}$.

In the past, most researchers directly used the curves of UPC versus embedment depth instead of load-displacement curves in comparing numerical results with model tests. To have a deeper insight of the load-displacement response of anchors, the whole pullout process of the anchor from the initial embedment depth to the mudline is simulated in the present work and compared with model tests. Figure 8 presents a comparison of normalized load-depth curves during the whole pullout process between the numerical results at the rigidity index $E / s_{u}=100$ and the model test data under $50 \mathrm{~g}$, in which $\mathrm{Q}$ denotes the calculated resisting force and $s_{u, 0}$ denotes the soil strength at the initial embedment depth of the anchor. As shown, both the present study and the model tests have the same tendency in the load-displacement curves. The resisting forces increase rapidly to reach the maximum capacity and then drop immediately. At the mudline, the resisting force almost reduces to zero in the numerical study since the strength is zero at the mudline. The small residual resistance at the mudline in experiments might be due to the weight of upheaval soil.

As reported by Chen et al. (2014), in the centrifuge tests under different gravity accelerations, the configuration of load-displacement curves would be different, while the maximum resisting forces remain the same. Figure 9 shows the normalized load-depth curves calculated at the embedment ratio of 2.86 and obtained from model tests under 50 and $100 \mathrm{~g}$. As can be seen that, the numerical result agrees better with the model test curve under $100 \mathrm{~g}$ in terms of the configuration of the load-depth curve. Anyway, the peak values, i.e., the maximum resisting forces, are very close.

\section{(3) Circular anchor in linear clay}

The numerical modeling is based on the centrifuge tests conducted by Wang et al. (2013) at an acceleration of $100 \mathrm{~g}$. Cases of single-plate helical anchors are modeled. The helix is simplified as a regular circular plate anchor with diameter $(D)$ of 2.4 $\mathrm{m}$ and thickness $(t)$ of $0.03 \mathrm{D}$. The remolded undrained shear strength of the soil is represented as $(\mathrm{kPa})$ and the saturated unit weight is $16 \mathrm{kN} / \mathrm{m}^{3}$. Since the rigidity index $E / s_{u}$ was unknown in the original model tests, $E / s_{u}=100,300$ and 500 are assigned for the numerical modeling. Cases with $H / D=2.6$ and 4.1 are examined. Through a parameter study on mesh size and pullout rate, the minimum mesh size of soil around the moving path of anchor is chosen as 0.021 $\mathrm{D}$ and the pullout rate of anchor is chosen as $0.021 \mathrm{D}$ $\mathrm{m} / \mathrm{s}$. Other conditions including the yield criterion, values of $K_{0}$ and the Poisson's ratio, the contact between soil and anchor and the boundary condition are all the same with the previous model.

The comparison of the bearing capacity factor between centrifuge tests and numerical modeling is listed in Table 1. Note that Wang et al. (2013) adopted the same criterion for determining the UPC as in the present study. As observed that, values of the UPC calculated by numerical modeling are smaller than the test data. The maximum difference between model tests and present modeling is $19.1 \%$ with the rigidity index $E / s_{u}=100$ at the embedment ratios of both 2.6 and 4.1, while the minimum difference is $11.5 \%$ with the rigidity index $E / s_{u}=500$ at the embedment ratio 2.6. As reported by Wang et al. (2013), the difference may be due to variations in soil disturbance and soil strength. The remolded strength was based on four T-bar tests after the pullout tests of helical anchors with certain unstated number of plates and a typical result was presented. The soil disturbance during the installation of single-plate helical anchor was less than the installation of multi- 
plate anchors. Therefore, the measured soil strength may be conservative for single-plate anchors.

The whole pullout processes of the anchor with the rigidity index $E / s_{u}=500$ are computed. The loaddepth curves from the centrifuge tests and the present modeling are presented in Fig. 10. It can be observed that the numerical results have a similar tendency as the centrifuge test data. The resisting force increases rapidly at the beginning and reaches the UPC. After that, it decreases gradually in the numerical modeling, while in the centrifuge tests it remains stable for a while then decreases gradually. The slopes of curves in centrifuge tests are close to those in the numerical modeling. At the mudline, a relatively large resisting force can be found in both the numerical modeling and the centrifuge tests. This is mainly because the undrained shear strength at the mudline is still quite large, which was estimated at $16 \mathrm{kPa}$.

Table 1. Comparison of the bearing capacity factor between centrifuge tests and numerical modeling

\begin{tabular}{|c|c|c|c|c|c|c|c|}
\hline \multirow{2}{*}{$\begin{array}{l}\text { Embedment } \\
\text { ratio (H/D) }\end{array}$} & \multirow[b]{2}{*}{ Measured } & \multicolumn{3}{|l|}{ Calculated } & \multicolumn{3}{|c|}{$\begin{array}{l}\text { Difference between the } \\
\text { measured and CEL (\%) }\end{array}$} \\
\hline & & $\mathrm{E}=100 \mathrm{~s}_{\mathrm{u}}$ & $\mathrm{E}=300 \mathrm{~s}_{\mathrm{u}}$ & $\mathrm{E}=500 \mathrm{~s}_{\mathrm{u}}$ & $\mathrm{E}=100 \mathrm{~s}_{\mathrm{u}}$ & $\mathrm{E}=300 \mathrm{~s}_{\mathrm{u}}$ & $\mathrm{E}=500 \mathrm{~s}_{\mathrm{u}}$ \\
\hline 2.6 & 13.62 & 11.02 & 11.82 & 12.06 & -19.1 & -13.2 & -11.5 \\
\hline 4.1 & 14.96 & 12.10 & 12.54 & 12.72 & -19.1 & -16.2 & -14.9 \\
\hline
\end{tabular}

a

b

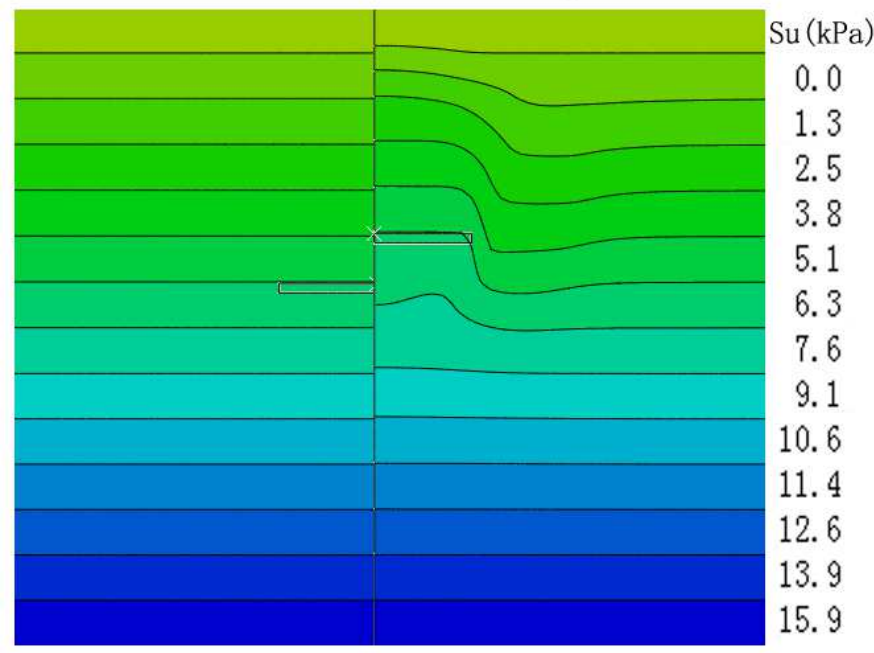

0.0
1.3
2.5
3.8
5.1
6.3
7.6
9.1
10.6
11.4
12.6
13.9
15.9

Fig. 6. The contour of linear soil strength during the anchor pullout process

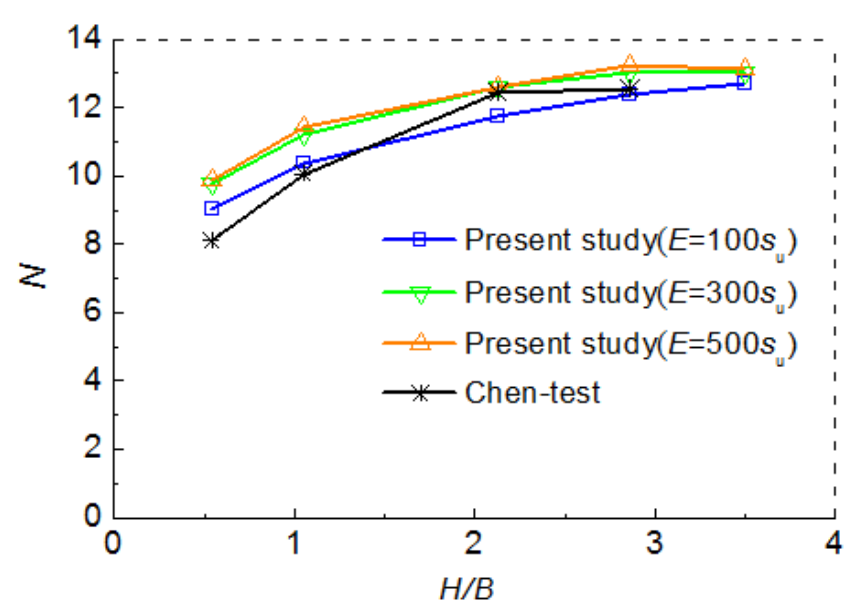

Fig. 7. Comparison of the bearing capacity factor of the square anchor in linear clay 


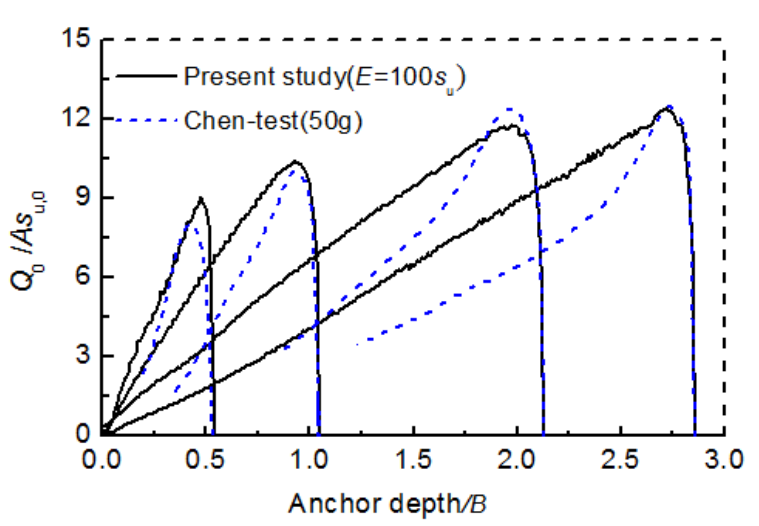

Fig. 8. Load-depth curves of anchors compared with model tests under $50 \mathrm{~g}$

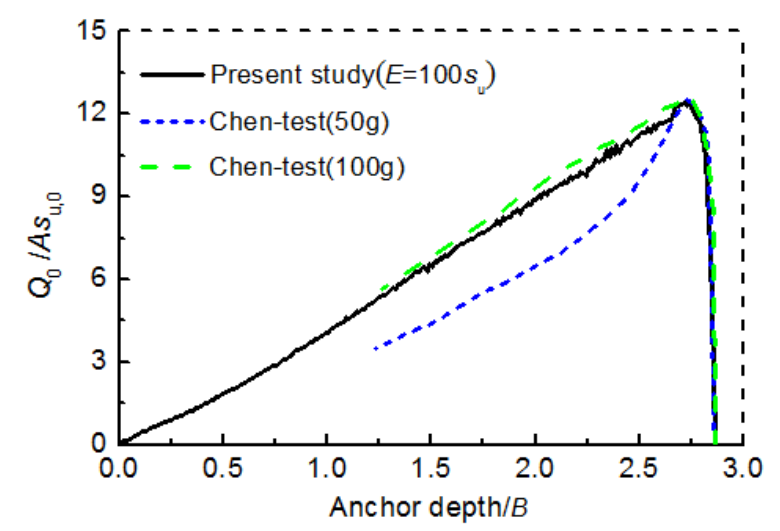

Fig. 9.Load-depth curves of anchors compared with model tests under 50 and $100 \mathrm{~g}$

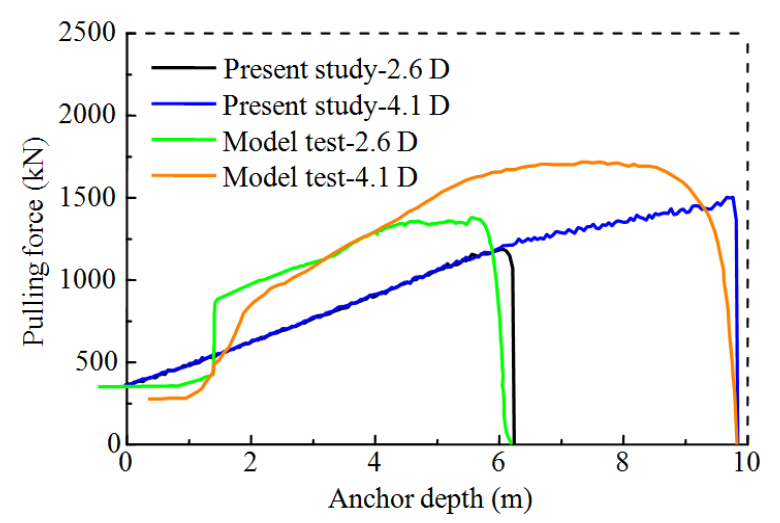

Fig. 10. Comparison of the load-depth curve between present modeling and centrifuge tests

\section{Applications}

The above validation work confirms the reliability and veracity of the large deformation finite element analysis and the criterion for determining the UPC. In this section, they are applied to analyzing rectangular anchors with different aspect ratios and circular anchors in both uniform and linear clays. In numerical study, many researchers directly calculated the UPC of anchors in weightless soil and then extended this solution to the soil with self weight through a function related to the soil overburden pressure, as reported by Rowe (1978; Das and Singh, 1994; Merifield et al., 2005; Chen et al., 2013). Considering that the unit weight of soil is different from one another, the UPC of anchors in weightless soil is also analyzed in the present work.

Rectangular anchors with the aspect ratio $(L / B)$ varying from 1 to 6 and $\infty$ are analyzed, where $L$ denotes the length of the rectangular anchor. The anchor with aspect ratio $L / B=\infty$ is regarded as the strip anchor and approximated as a plane strain problem using a one-element thick mesh. The anchor has the width $(B)$ of $3 \mathrm{~m}$ and the thickness $(t)$ of $0.05 \mathrm{~B}$. For circular anchor, the diameter of anchor $D$ is $3.38 \mathrm{~m}$ and the thickness is $0.05 \mathrm{D}$. The undrained shear strength $\left(s_{u}\right)$ is $10 \mathrm{kPa}$ for uniform clay, while for linear clay is represented as $s_{u}=z(\mathrm{kPa})$. The gravity force is set to zero. The soil rigidity index $E / s_{u}$ is assigned as 500 . Cases with H/B varying from 1 to 10 are examined. Based on parametric studies, the minimum mesh size of soil around the moving path and the pullout rate of anchor are selected as $0.025 \mathrm{~B}$ (or $0.025 \mathrm{D}$ ) and $0.04 \mathrm{~B}$ (or $0.04 \mathrm{D}$ ) $\mathrm{m} / \mathrm{s}$, respectively. The yield criterion, $K_{0}$, the Poisson's ratio, the contact between soil and anchor and the boundary condition are all identical with those adopted in the previous model.

\section{Square Anchor in Uniform Clay}

In the case of $L / B=1$ of rectangular anchors, the anchor is a square plate anchor. The bearing capacity factors for the square plate anchor in uniform soil at different embedment ratios are calculated and compared with the numerical results from other researchers, as presented in Fig. 11.

Chen et al. (2013) analyzed the UPC of square anchors in weightless soil using the same large deformation technique CEL. The present results are a bit higher than those of Chen et al. (2013). The main reason is that "the plateau criterion" was adopted in demining the UPC in their study. Wang et al. (2009) used the same criterion as in the present modeling while utilized a different large deformation technique RITSS. A slight difference between the results of Wang et al. (2009) and the present study can be observed, probably attributed to different large deformation techniques employed.

For embedment ratios less than 5, present results are close to those by Merifield et al. (2005) based on lower bound limit analysis and $\mathrm{Yu}$ (2000) based on cavity expansion theory. For embedment ratios higher than 5 , the results of large deformation analysis are all smaller than the results from other methods. The cavity theory proposed by $\mathrm{Yu}$ (2000) is based on 
small strain analysis. The limit analysis employed by Merifield et al. (2005) considered neither the initial condition of stress nor the deformation process of soil and directly solved the equilibrium equations of stress. In other words, Merifield et al. (2005) assumed the failure of anchor happened in its initial position. As reported by Wang et al. (2009), both theories would inevitably overestimate the capacity of the deeply embedded anchor since it fails at a shallower depth than its initial position.

\section{Rectangular Anchor in Clay}

Figure 12 presents the bearing capacity factors of rectangular anchors versus embedment ratios in uniform clay, which indicates that with growing embedment depth, the UPC of anchors gradually increases and ultimately reaches a limiting value. The anchor with a larger aspect ratio achieves the limiting value at a deeper embedment depth. A critical embedment ratio can be defined to differentiate the shallow or deep embedment, which is the embedment ratio at which the anchor just reaches the limiting value. It is observed that the critical embedment ratio increases with increasing aspect ratio. The critical embedment ratio is 6 for the square anchor, while for rectangular anchors with the aspect ratio larger than 4 , the critical embedment ratio cannot be reached within the embedment depth $10 \mathrm{~B}$.

It is also observed that the limiting values are different for different aspect ratios and generally decrease with increasing aspect ratio. However, the UPC of the anchor with the aspect ratio 5 is higher than the aspect ratio 6 with the maximum difference of $3.78 \%$. It is then anticipated that the UPC would not have an evident reduction if the aspect ratio is higher than 6 . Therefore, the anchor with the aspect ratio larger than 6 could be considered as a strip anchor. However, the maximum difference of the UPC between the strip anchor and the rectangular anchor with the aspect ratio 6 is $34.96 \%$. This may be attributed to the assumption of the plane strain condition for the strip anchor, since the contribution to the resisting force from both sides along the length direction is neglected.

Figure 13 presents the bearing capacity factors of rectangular anchors versus embedment ratios in linear clay, which indicates that with growing embedment depth, the UPC of anchors gradually increases but cannot reach a limiting value within the embedment depth $10 \mathrm{~B}$ except in the case $L / B=1$. With increasing aspect ratio, the UPC of anchors gradually decreases. However, if the aspect ratio is higher than 4, the reduction in UPC is not evident. The UPC of the anchor with the aspect ratio 5 is higher than the aspect ratio 6 with a maximum difference of $3.79 \%$. Hence, the rectangular anchor with the aspect ratio higher than 4 in linear clay could be considered as a strip anchor.

Compared with the uniform clay, the UPC of anchors in linear clay, whose strength at the seafloor $(z=0)$ is 0 , is much lower. In linear clay, the anchor always approaches to the weaker soil during the pullout process so that the resistance force fails to be fully developed. Moreover, the critical embedment ratio in linear clay is larger than that in uniform clay. In uniform clay, the critical embedment ratio of square anchor is 6 , while it is 9 in linear clay.

\section{Circular Anchor in Clay}

As illustrated in Fig. 14, in both uniform and linear clays, the UPC of the circular anchor is a little higher than that of the square anchor. For example, the maximum difference in uniform clay is $7.84 \%$. In linear clay, the UPC of the circular anchor is very close to that of the square anchor at the embedment depth larger than $7 \mathrm{~B}$.

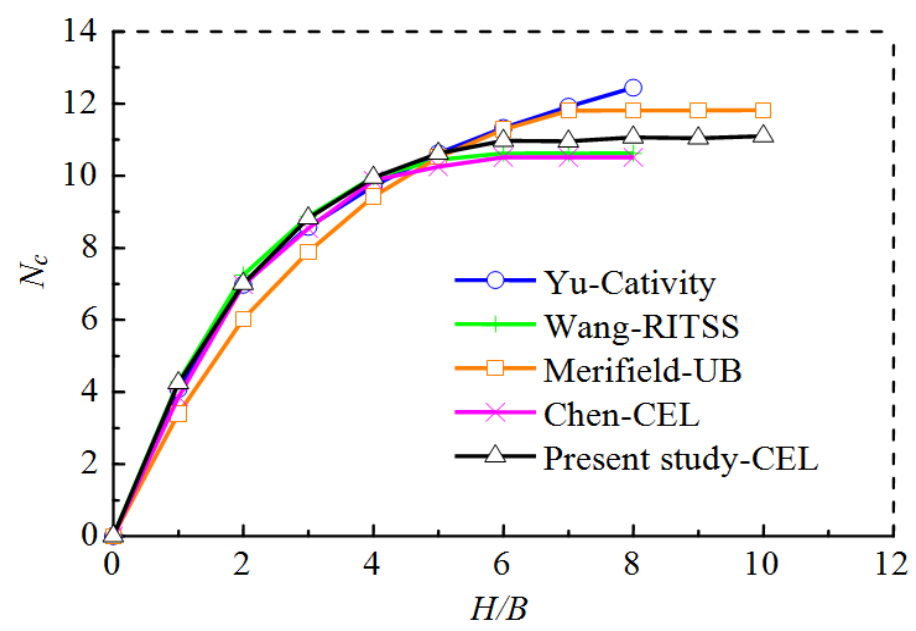

Fig. 11. Comparison of the bearing capacity factor of the square anchor in uniform clay 


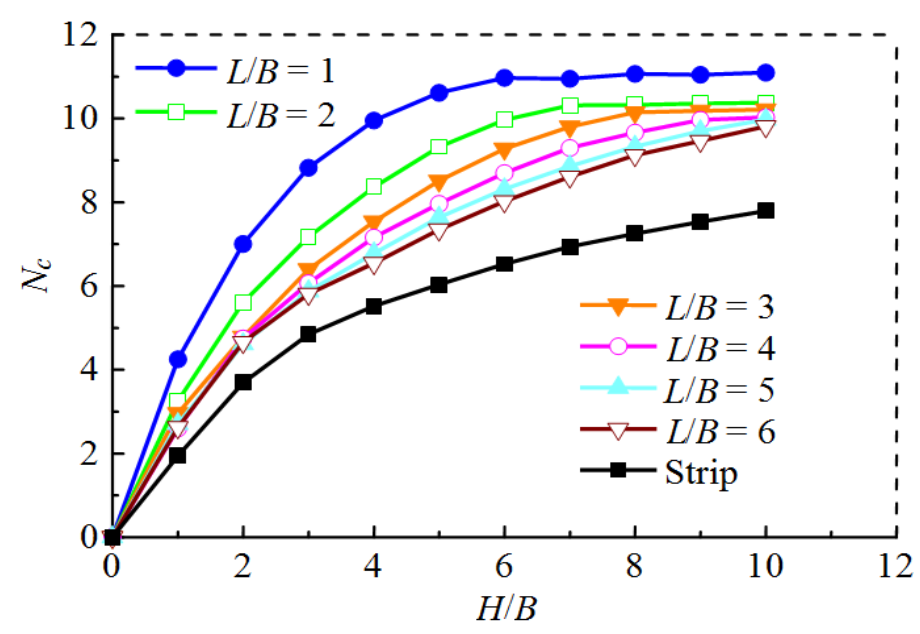

Fig. 12. Bearing capacity factors of rectangular anchors in uniform clay

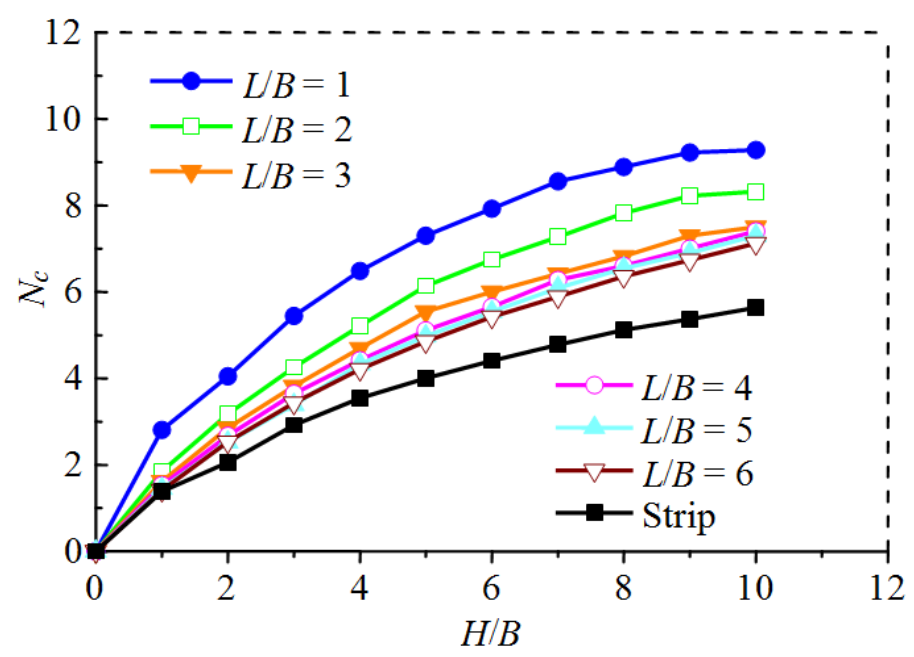

Fig. 13. Bearing capacity factors of rectangular anchors in linear clay

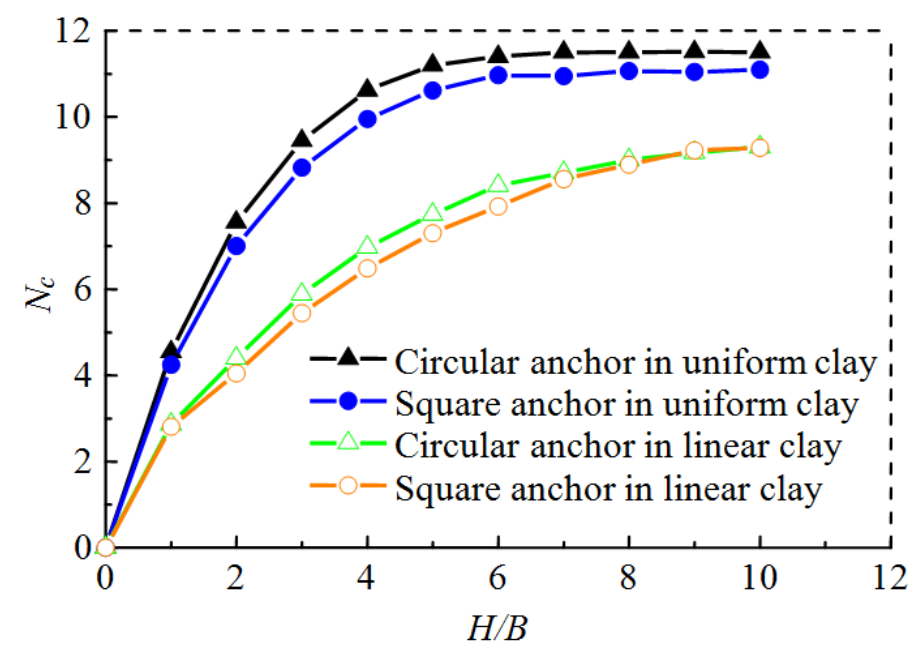

Fig. 14. Comparison of the bearing capacity factor between circular and square anchors 
In uniform clay, the critical embedment ratio for the circular anchor is about 5 , which is less than the square anchor. In linear clay, the critical embedment ratio cannot be reached within the embedment depth $10 \mathrm{~B}$.

\section{Conclusion}

The UPC is an important index in designing and analyzing plate anchors. However, there are still divergences in determining the UPC and specific studies are rare. Hence, further investigations are needed to clarify this basic but important topic. The present work emphasizes on the criterion for determining the UPC of plate anchors in clay. After a review of current studies on this topic, the criterion for determining the UPC based on large deformation finite element analysis is recommended. This criterion is validated firstly by model tests and then applied to rectangular and circular plate anchors at different embedment ratios in both uniform and linear clays to calculate bearing capacity factors.

The proposed criterion conforms to two rules. The first, a deterministic value of UPC can be obtained by using the criterion. The second, the criterion must be rational which means that it implies clear and right physical meaning and can be generally applied. The maximum resistance criterion based on large deformation analysis is definitely recommended, in which the coupled Eulerian-Lagrangian (CEL) technique is adopted in the present work.

Although the large deformation analysis can well simulate the whole pullout process and obtain a complete load-displacement curve of plate anchors, the compensation is that more computing time is needed compared with small deformation methods. In practice, to obtain a complete load-displacement curve is not always necessary. To analyze the UPC or bearing capacity factor, to acquire the peak value of the loaddisplacement curve is enough. Hence, we need only to calculate to the peak value and this may save $10-50 \%$ of the total time for anchors in uniform soil and $50-90 \%$ for anchors in linear soil.

Although the present work aims at plate anchors in clay, the proposed criterion for determining the UPC may apply to other types of structures embedded in other soils. However, the applicability of the proposed criterion needs to be examined in the future.

\section{Acknowledgement}

Financial support from the Key Project of Tianjin Research Program of Application Foundation and Advanced Technology (14JCZDJC39900) and the National Natural Science Foundation of China (Grant nos. 51179124 and 50979070) is greatly acknowledged.

\section{Author's Contributions}

Haixiao Liu: Designed the research plan and goal. Made considerable contributions to conception, the writing and revising of the manuscript.

Fangmei Su: Contributed to the literature review and conducted all the numerical studies.

Zhou Li: Participated in the literature review and data-analysis. Contributed to the drafting and revising of the manuscript

\section{Ethics}

We guarantee the truth of all data and the originality of this paper.

\section{References}

Boushehrian, A.H., N. Hataf and A. Ghahramani, 2009. Numerical study of cyclic behavior of shallow foundations on sand reinforced with geogrid and grid-anchor. World Acad. Sci. Eng. Technol., 3: 593-596.

Chen, J., K.K. Tho, C.F. Leung and Y.K. Chow, 2014. Centrifuge model study on vertical pullout behaviour of square plate anchors in normally consolidated clay. Proceedings of the Offshore Technology Conference-Asia, Mar. 25-28, Offshore Technology Conference, Kualu Lumpur, Malaysia.

Chen, Z., K.K. Tho, C.F. Leung and Y.K. Chow, 2013. Influence of overburden pressure and soil rigidity on uplift behavior of square plate anchor in uniform clay. Comput. Geotechn., 52: 71-81.

DOI: 10.1016/j.compgeo.2013.04.002

Das, B.M. and G. Singh, 1994. Uplift capacity of plate anchors in clay. Proceedings of the 4th International Offshore and Polar Engineering Conference, (EPC' 94), ISOPE, Osaka, Japan.

Fahmy, A.M., J.R. de Bruyn and T.A. Newson, 2013. Numerical investigation of the inclined pullout behavior of anchors embedded in clay. Geotechn. Geol. Eng., 31: 1525-1542.

DOI: 10.1007/s10706-013-9676-9

Ghaly, A., 1997. Load-displacement prediction for horizontally loaded vertical plates. J. Geotechn. Geoenviron. Eng., 123: 74-76. DOI: 10.1061/(ASCE)1090-0241(1997)123:1(74)

Ghaly, A., A. Hanna and M. Hanna, 1991. Uplift behavior of screw anchors in sand. I: Dry sand. J. Geotechn. Eng., 117: 773-793. DOI: 10.1061/(ASCE)0733-9410(1991)117:5(773)

Hanna, A., T. Ayadat and M. Sabry, 2007. Pullout resistance of single vertical shallow helical and plate anchors in sand. Geotechn. Geol. Eng., 25: 559-573. DOI: $10.1007 / \mathrm{s} 10706-007-9129-4$ 
Merifield, R.S., A.V. Lyamin and S.W. Sloan, 2005. Stability of inclined strip anchors in purely cohesive soil. J. Geotechn. Geoenviron. Eng., 131: 792-799. DOI: 10.1061/(ASCE)1090-0241(2005)131:6(792)

Mistri, B. and B. Singh, 2011. Pullout behavior of plate anchors in cohesive soils. Electronic J. Geotechn. Eng., 16: 1173-1184.

Neeley, W.J., J.G. Stuart and J. Graham, 1973. Failure loads of vertical anchor plates in sand. J. Soil Mechan. Foundat. Divis., 99: 669-685. http://cedb.asce.org/cgi/WWWdisplay.cgi?20206

O’Neill, M.P., M.F. Bransby, M.F. Randolph, 2003. Drag anchor fluke soil interaction in clays. Canad. Geotechn. J., 40: 78-94. DOI: 10.1139/t02-096

Rowe, R.K., 1978. Soil structure interaction analysis and its application to the prediction of anchor plate behaviour. PhD. thesis, University of Sydney, Australia.

Rowe, R.K. and E.H. Davis, 1982. The behaviour of anchor plates in clay. Geotechnique, 32: 9-23. DOI: $10.1680 /$ geot.1982.32.1.9

Sahoo, J.P. and J. Kumar, 2013. Vertical uplift resistance of two interfering horizontal anchors in clay. J. Geotechn. Geoenviron. Eng., 140: 06013007-06013007. DOI: 10.1061/(ASCE)GT.1943-5606.0001073
Singh, S.P. and S.V. Ramaswamy, 2008. Effect of shape on holding capacity of plate anchors buried in soft soil. Geomechan. Geoeng., 3: 145-154. DOI: $10.1080 / 17486020802126875$

Song, Z., Y. Hu and M.F. Randolph, 2008. Numerical simulation of vertical pullout of plate anchors in clay. J. Geotechn. Geoenviron. Eng., 134: 866-875. DOI: 10.1061/(ASCE)1090-0241(2008)134:6(866)

Vesic, A.S., 1973. Analysis of ultimate loads of shallow foundations. J. Soil. Mech. Found. Eng., 99: 45-76.

Wang, D., Y.X. Hu and M.F. Randolph, 2009. Threedimensional large deformation finite-element analysis of plate anchors in uniform clay. J. Geotechn. Geoenviron. Eng., 136: 355-365. DOI: 10.1061/(ASCE)GT.1943-5606.0000210

Wang, D., R.S. Merifield and C. Gaudin, 2013. Uplift behaviour of helical anchors in clay. Canad. Geotechn. J., 50: 575-584. DOI: 10.1139/cgj-2012-0350

Yang, M., J.D. Murff and C.P. Aubeny, 2010. Undrained capacity of plate anchors under general loading. J. Geotechn. Geoenviron. Eng., 136: 1383-1393. DOI: 10.1061/(ASCE)GT.1943-5606.0000343

$\mathrm{Yu}$, H.S., 2000. Cavity Expansion Methods in Geomechanics. 1st Edn., Springer Science and Business Media, ISBN-10: 0412799901, pp: 385. 